\title{
Self-similar evolution of wind-blown bubbles with mass-loading by conductive evaporation
}

\author{
J. M. Pittard, J. E. Dyson, and T. W. Hartquist \\ Department of Physics \& Astronomy, The University of Leeds, Woodhouse Lane, Leeds, LS2 9JT, UK
}

Received 13 November 2000 / Accepted 18 December 2000

\begin{abstract}
We present similarity solutions for adiabatic bubbles that are blown by winds having time dependent mechanical luminosities and that are each mass-loaded at a rate per unit volume proportional to $T^{5 / 2} r^{\lambda}$, where $T$ is the temperature, $r$ is the distance from the bubble center, and $\lambda$ is a constant. In the limit of little mass loading a similarity solution found by Dyson (1973) for expansion into a smooth ambient medium is recovered. For solutions that give the mass of swept-up ambient gas to be less than the sums of the masses of the wind and the evaporated material, $\lambda \gtrsim 4$. The Mach number in a shocked mass-loaded wind shows a radial dependence that varies qualitatively from solution to solution. In some cases it is everywhere less than unity in the frame of the clumps being evaporated, while in others it is everywhere greater than unity. In some solutions the mass-loaded shocked wind undergoes one or two sonic transitions in the clump frame. Maximum possible values of the ratio of evaporated mass to stellar wind mass are found as a consequence of the evaporation rates dependence on temperature and the lowering of the temperature by mass-loading. Mass-loading tends to reduce the emissivity in the interior of the bubble relative to its limb, whilst simultaneously increasing the central temperature relative to the limb temperature.
\end{abstract}

Key words. hydrodynamics - shock waves - stars: mass-loss - ISM: bubbles - galaxies: active

\section{Introduction}

Many authors have pointed out that mass pick-up from embedded clumps can significantly modify the structures of a wide variety of astrophysical flows. These include, for example, McKee \& Ostriker (1977) in their theory of the interstellar medium, Hartquist et al. (1986) in the study of wind blown nebulae, and Chièze \& Lazareff (1981); Cowie et al. (1981) and Dyson \& Hartquist (1987) in the study of supernova remnants (SNRs).

Observations of wind-blown bubbles (WBBs) (e.g. Smith et al.1984; Meaburn et al.1991) have led to the conclusion that the interactions of the winds with clumps of stellar material ejected during prior stages of massloss greatly affect the structure and evolution of the bubbles (see Williams et al.1995 and references therein). Consequently, a number of numerical investigations of this process have been conducted. Detailed one-dimensional, time dependent hydrodynamic models of specific WBBs have been constructed by Arthur et al. $(1993,1994)$ and Arthur et al. (1996). Numerical solutions for the Mach numbers of steady mass-loaded winds have been presented by Williams et al. (1995), and studies of steady hydromag-

Send offprint requests to: J. M. Pittard, e-mail: jmp@ast.leeds.ac.uk netic mass-loaded winds have been performed by Williams et al. (1999).

In this paper we derive similarity solutions for the structures and evolution of mass-loaded WBBs. This work complements that on similarity solutions for SNRs obtained by Chièze \& Lazareff (1981) and Dyson \& Hartquist (1987). Here we consider a constant rate of energy input, $\dot{E}$, rather than a constant total energy, and assume that the mass-loading occurs due to conductively driven evaporation. The solutions are potentially relevant to WBBs created by a fast wind interacting with a clumpy AGB superwind, by the wind of a young high-mass star interacting with surrounding molecular material, and the wind of an active galactic nucleus impacting its environment.

\section{Similarity solutions}

\subsection{Overview of assumptions}

The evaporation of spherical clouds by hot gas has been analyzed by Cowie \& McKee (1977) and McKee \& Cowie (1977). If the mean free path for electron-electron energy change in the ambient gas is less than about half of the radius of a clump, the mass evaporation rate from an individual clump scales as $T^{5 / 2}$, where $T$ is the temperature of the material surrounding the clump. For a typical jump 
of $1000 \mathrm{~km} \mathrm{~s}^{-1}$ at the reverse shock (corresponding to a post-shock temperature of $T \approx 1.410^{7} \mathrm{~K}$ ), and a clump electron number density of $n_{\mathrm{e}} \approx 10^{4}$, the mean free path for electron energy exchange is $\lambda \approx 510^{15} \mathrm{~cm}$. This is somewhat larger than the width of a clump in a planetary nebula (e.g. Meaburn et al. 1998) but many clumps have long tails along which the magnetic field might be expected to be aligned; thus, the assumption of unsaturated conduction with a $T^{5 / 2}$ temperature dependence of $\dot{\rho}$, the mass evaporation rate per unit volume, should be reasonable throughout most of the mass-loading region if mass-loading is significant.

We also assume that the clumps are stationary with respect to the star, and that they are also cold and dense. Therefore the clumps have neither kinetic or internal energy, and the picked-up mass is thus injected at zero velocity. The stripped mass then acquires momentum and energy from the general more tenuous flow. Hence the only source term in the hydrodynamic equations is $\dot{\rho}$. In addition we assume that at any given point the injected mass reaches the general flow velocity and temperature instantaneously i.e. that the characteristic scale length of the mixing region is much smaller than the dimensions of the bubble. We also assume that the clumps are sufficiently numerous that they can be considered continuously distributed such that a hydrodynamic treatment applies. We suppose that conductive energy transport can be neglected in the energy equation describing the global evolution of the WBB. The WBBs are assumed to evolve adiabatically, whilst the swept-up interclump medium is assumed to catastrophically cool into a thin dense shell. The clumps are assumed to pass through this shell without any interaction, and then on into the region of shocked stellar wind. The unshocked stellar wind is assumed to have a flow speed much greater than its thermal speed. Hence its thermal energy is negligible with respect to its kinetic energy, and since no mass-loading occurs in this region, we ignore the energy equation for this part of the flow. Finally we assume that any external forces, such as gravity or radiation pressure, are negligible.

A schematic of the flow topology is shown in Fig. 1. The hydrodynamical equations for the SSW region are:

$\frac{\partial \rho}{\partial t}+\nabla \cdot(\rho u)=\dot{\rho}$

$\frac{\partial(\rho u)}{\partial t}+\nabla \cdot\left(\rho u^{2}\right)+\frac{\partial P}{\partial r}=0$

$\frac{\partial\left(\frac{1}{2} \rho u^{2}+\varepsilon\right)}{\partial t}+\nabla \cdot\left(\rho u\left(\frac{1}{2} u^{2}+\frac{5}{3} \frac{\varepsilon}{\rho}\right)\right)=0$

where the symbols have their usual meanings. For conductively induced mass-loading, the evaporation of the clumps only occurs in the SSW region. In the USW region we assume no interaction between the clumps and the fast stellar wind.

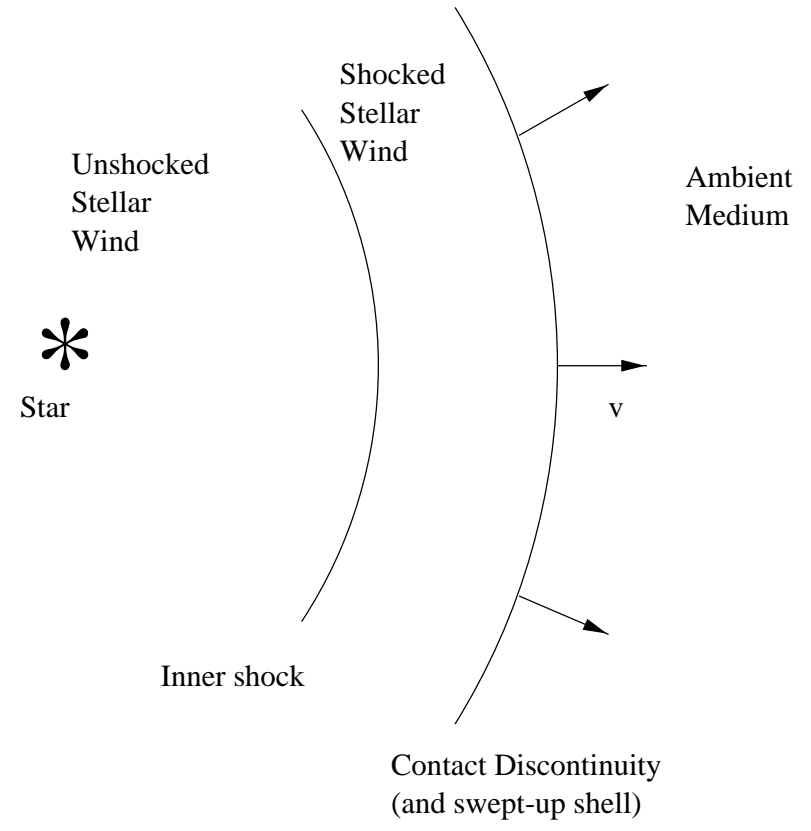

Fig. 1. Schematic of the flow structure in our solutions. The central star blows out a bubble into the surrounding medium which is characterized by a region of unshocked stellar wind (USW), a region of shocked stellar wind (SSW), and a dense shell of swept-up material (SUM). We assume that the shocked ambient medium quickly radiates all of its internal energy, whilst the cooling time of the SSW is assumed to be much longer than the current age of the bubble. Mass-loading only occurs in the SSW

\subsection{The similarity variables}

Let the interclump medium have a density of the form $\rho_{\text {ic }}=\rho_{0} r^{\beta}$, and let us consider the case where the massloading rate is also radially dependent: $\dot{\rho}=Q(\varepsilon / \rho)^{5 / 2} r^{\lambda}$. The problem as its stands has five parameters with independent dimensions, namely $r, t, Q, \rho_{0}$, and the rate of energy input by the stellar wind $\dot{E}$. The solution inside the bubble depends on $r, t, Q$, and $\dot{E}$, whilst the solution ahead of the contact discontinuity depends on $r, t$, $\rho_{0}$, and $\dot{E}$. Inside the bubble we can construct a variable $r=x \dot{E}^{u} t^{v} Q^{w}$ with a dimension of length. Outside the bubble we construct a similar variable $r^{\prime}=x^{\prime} \dot{E}^{x} t^{y} \rho_{0}^{z}$. Both $x$ and $x^{\prime}$ are dimensionless. For a similarity solution to exist $v=y$, which demands that $\lambda=(5+7 \beta) / 3$. This reduces the number of dimensional parameters to $4: r, t$, $Q$, and $\dot{E}$. The solution may then be expressed in terms of the dimensionless similarity variables $x, f(x), g(x)$, and $h(x)$ where:

$r=x Q^{-1 /(10+\lambda)} \dot{E}^{1 /(10+\lambda)} t^{7 /(10+\lambda)}$
$\rho=Q^{5 /(10+\lambda)} \dot{E}^{(5+\lambda) /(10+\lambda)} t^{-(5-3 \lambda) /(10+\lambda)} f(x)$
$u=Q^{-1 /(10+\lambda)} \dot{E}^{1 /(10+\lambda)} t^{-(3+\lambda) /(10+\lambda)} g(x)$
$\varepsilon=Q^{3 /(10+\lambda)} \dot{E}^{(7+\lambda) /(10+\lambda)} t^{-(11-\lambda) /(10+\lambda)} h(x)$.

The hydrodynamic equations for the region of shocked stellar wind become the following set of coupled ordinary 
differential equations:

$$
\begin{aligned}
\left(g-\frac{7 x}{10+\lambda}\right) f^{\prime}+f g^{\prime} & +\frac{2 f g}{x}-\frac{5-3 \lambda}{10+\lambda} f \\
& -\left(\frac{h}{f}\right)^{5 / 2} x^{\lambda}=0 \\
\left(g-\frac{7 x}{10+\lambda}\right) g^{\prime}+\frac{2}{3 f} h^{\prime} & -\frac{3+\lambda}{10+\lambda} g \\
+ & \frac{g}{f}\left(\frac{h}{f}\right)^{5 / 2} x^{\lambda}=0 \\
\left(g-\frac{7 x}{10+\lambda}\right) h^{\prime}+\frac{5}{3} h g^{\prime} & -\frac{11-\lambda}{10+\lambda} h \\
& +\frac{10}{3 x} h g-\frac{g^{2}}{2}\left(\frac{h}{f}\right)^{5 / 2} x^{\lambda}=0
\end{aligned}
$$

where a prime denotes derivation with respect to $x$. It is simple to rearrange these equations to find $f^{\prime}, g^{\prime}$, and $h^{\prime}$.

\subsubsection{Boundary conditions}

We start our integration at a point very close to the star. As $r \rightarrow 0,4 \pi r^{2} \rho v \rightarrow \dot{M}_{\mathrm{c}}$, where $\dot{M}_{\mathrm{c}}$ is the current rate of mass-loss of the $\operatorname{star}(\dot{M}$ is a function of time if $\lambda \neq-3)$. Substituting the similarity variables we obtain:

$\dot{M}_{\mathrm{c}}=\lim _{x \rightarrow 0} 4 \pi x^{2} f g \Xi$

where $\Xi=Q^{2 /(10+\lambda)} \dot{E}^{(8+\lambda) /(10+\lambda)} t^{(6+2 \lambda) /(10+\lambda)}$. We define $\phi$, such that

$\phi=\frac{\dot{M}_{\mathrm{c}}}{\Xi}=\lim _{x \rightarrow 0} 4 \pi x^{2} f g$

which is a measure of the ratio of evaporated mass to wind mass. We also require that as $r \rightarrow 0,2 \pi r^{2} \rho v^{3} \rightarrow \dot{E}$. i.e. that

$\dot{E}=\lim _{x \rightarrow 0} 2 \pi x^{2} f g^{3} \dot{E}$.

Cancelling the $\dot{E}$ 's and substituting into Eq. (12) we obtain the values of $f$ and $g$ at $x=\Delta x$, the inner boundary of our integration: $g=\sqrt{2 / \phi}, f=1 /\left(2 \pi \Delta x^{2} g^{3}\right)$.

The inner shock is positioned at $x=x_{\text {is }}$. Once the integration has proceeded to this point the standard RankineHugoniot jump conditions across a discontinuity $\Sigma$ must be satisfied:

$[\rho u]_{\Sigma}=0 ; \quad\left[\rho v^{2}+p\right]_{\Sigma}=0 ; \quad\left[\frac{1}{2} v^{2}+\frac{\gamma}{\gamma-1} \frac{p}{\rho}\right]_{\Sigma}=0$

The pre-shock flow has an effectively infinite Mach number and the strong shock jump conditions apply. If the postshock values for the density and the velocity are given by $f_{2}$ and $g_{2}$, the Rankine-Hugoniot conditions for a $\gamma=5 / 3$ gas specify that the post-shock value of the thermal energy density, $h_{2}$, is:

$h_{2}=\frac{9}{2} f_{2}\left(g_{2}-g_{\mathrm{s}}\right)^{2}$ where $g_{\mathrm{s}}=7 x_{\text {is }} /(10+\lambda)$ is the velocity of the inner shock relative to the star.

Once the post-shock conditions have been calculated, integration proceeds until the contact discontinuity (CD) is reached. This occurs once the flow velocity is equal to the coordinate velocity (i.e. $v=\mathrm{d} R / \mathrm{d} t$ ), at which point $g\left(x=x_{\mathrm{cd}}\right)=7 x /(10+\lambda)$ is satisfied. At the CD, we must also satisfy conservation of momentum. This requires that the pressure inside the bubble is equal to the impulse on the swept up shell:

$4 \pi r^{2} p=\frac{\mathrm{d}\left(M_{\mathrm{sh}} v\right)}{\mathrm{d} t}=v \frac{\mathrm{d} M_{\mathrm{sh}}}{\mathrm{d} t}+M_{\mathrm{sh}} \frac{\mathrm{d} v}{\mathrm{~d} t}$

where here $M_{\mathrm{sh}}$ is the mass of the swept-up shell $\left(M_{\mathrm{sh}}=\right.$ $\left.4 \pi \rho_{0} r_{\mathrm{cd}}^{3+\beta} /(3+\beta)\right)$, and $\mathrm{d} M_{\mathrm{sh}} / \mathrm{d} t=4 \pi r^{2} \rho v$. Equation (16) reduces to

$\rho_{0} r^{\beta} v^{2}+\frac{\rho_{0} r^{1+\beta}}{3+\beta} \frac{\mathrm{d} v}{\mathrm{~d} t}=p$.

We now construct a dimensionless constant, $\theta$, from $Q, \dot{E}$, and $\rho_{0}$ :

$\theta \equiv \frac{Q^{-1 /(10+\lambda)} \dot{E}^{1 /(10+\lambda)}}{\rho_{0}^{-1 /(5+\beta)} \dot{E}^{1 /(5+\beta)}}$

It provides a measure of the ratio of the evaporated mass to the swept-up mass. Inserting the similarity variables into Eq. (17) and substituting Eq. (18), we find after some rearrangement:

$\theta=\left(\frac{(\gamma-1) h}{x^{\beta}\left[g^{2}-\frac{3+\lambda}{(10+\lambda)(3+\beta)} x g\right]}\right)_{\mathrm{cd}}^{1 /(5+\beta)}$.

The value of $\theta$ is evaluated once the integration stops at the CD.

\subsection{Scale transformation and normalization}

The dimensionless Eqs. (8-10) (and their counterparts for the USW region) are invariant under the following transformation, which we shall call a normalization (cf. Chièze \& Lazareff 1981):

$$
\left.\begin{array}{l}
x \rightarrow \alpha x \\
f \rightarrow \alpha^{5+\lambda} f \\
g \rightarrow \alpha g \\
h \rightarrow \alpha^{7+\lambda} h
\end{array}\right\}
$$

These can be combined to obtain the normalizations for the mass, and for the kinetic and thermal energies, of the bubble:

$$
\begin{aligned}
m & =4 \pi \int f x^{2} \mathrm{~d} x & & {\left[\alpha^{8+\lambda}\right] } \\
k & =4 \pi \int \frac{1}{2} f g^{2} x^{2} \mathrm{~d} x & & {\left[\alpha^{10+\lambda}\right] } \\
i & =4 \pi \int h x^{2} \mathrm{~d} x & & {\left[\alpha^{10+\lambda}\right] . }
\end{aligned}
$$


The power of $\alpha$ in the square brackets indicates how each integral scales in a normalization. The full equations for the mass, kinetic and thermal energies, in the bubble are

$$
\begin{aligned}
M_{\mathrm{b}} & =4 \pi \alpha^{8+\lambda} \Xi t \int_{x=0}^{x_{\mathrm{cd}}} f x^{2} \mathrm{~d} x=\alpha^{8+\lambda} \frac{\dot{M}_{\mathrm{c}} t}{\phi} m \\
K E_{\mathrm{b}} & =2 \pi \alpha^{10+\lambda} \dot{E} t \int_{x=0}^{x_{\mathrm{cd}}} f g^{2} x^{2} \mathrm{~d} x=\alpha^{10+\lambda} \dot{E} t k \\
I E_{\mathrm{b}} & =4 \pi \alpha^{10+\lambda} \dot{E} t \int_{x=0}^{x_{\mathrm{cd}}} h x^{2} \mathrm{~d} x=\alpha^{10+\lambda} \dot{E} t i .
\end{aligned}
$$

The mass in the USW (SSW) region, $M_{\mathrm{usw}}\left(M_{\mathrm{ssw}}\right)$, is obtained through the replacement of $m$ in Eq. (24) with $m_{\mathrm{usw}}\left(m_{\mathrm{ssw}}\right)$, where the integral is evaluated from $x=0$ to $x=x_{\text {is }}$ (from $x=x_{\text {is }}$ to $x=x_{\mathrm{cd}}$ ). The total mass carried from the star by its wind is

$$
M_{\mathrm{w}}=\int \dot{M}(t) \mathrm{d} t=\frac{10+\lambda}{16+3 \lambda} \dot{M}_{\mathrm{c}} t .
$$

The degree of mass-loading in the bubble, $\Phi_{\mathrm{b}}$, can be measured by the ratio $M_{\mathrm{b}} / M_{\mathrm{w}}$. Starting with the expression for $M_{\mathrm{sh}}$, (cf. text following Eq. (16)), using Eq. (4) to express $r_{\text {cd }}$ in terms of $x_{\mathrm{cd}}$, employing Eq. (18) to rewrite $\rho_{0}$ in terms of $\theta$, and using Eq. (11) and the definition of $\Xi$, we find

$M_{\mathrm{sh}}=\frac{4 \pi}{3+\beta} \alpha^{8+\lambda} \theta^{5+\beta} x_{\mathrm{cd}}^{3+\beta} \frac{\dot{M}_{\mathrm{c}} t}{\phi}$.

The ratio of the swept-up mass in the shell to the bubble mass is hence:

$M_{\mathrm{sh}} / M_{\mathrm{b}}=\frac{4 \pi \theta^{5+\beta} x_{\mathrm{cd}}^{3+\beta}}{(3+\beta) m}$.

The kinetic energy of the shell is $K E_{\mathrm{sh}}=\frac{1}{2} M_{\mathrm{sh}} v_{\mathrm{cd}}^{2}$. Using Eqs. (28), (13), (20), and (8) through (10) we obtain

$K E_{\mathrm{sh}}=\frac{2 \pi}{3+\beta} \alpha^{10+\lambda} \dot{E} t \theta^{5+\beta} x_{\mathrm{cd}}^{3+\beta} g_{\mathrm{cd}}^{2}$.

We can also calculate the $p d V$ work done on the shocked interclump medium in compressing it into a negligibly thin shell. The initial energy per unit mass of the interclump gas immediately after passing through the forward shock is:

$E_{1}=\frac{1}{2} v_{1}^{2}+\frac{3}{2} \frac{p_{1}}{\rho_{1}}$

where here $v_{1}$ is the post-shock flow velocity $\left(=\frac{3}{4} v_{\mathrm{cd}}\right)$ and $p_{1}$ and $\rho_{1}$ are the post-shock pressure and density. If we assume that the pressure and velocity of the surrounding medium are both negligible, the post-shock pressure is $p_{1}=\frac{3}{4} \rho_{0} r_{\mathrm{cd}}^{\beta} v_{\mathrm{cd}}^{2}$. Substituting for $p_{1}$ into Eq. (31), we find that the sum of the kinetic and thermal energies per unit mass behind a strong shock is $E_{1}=\frac{9}{16} v_{\mathrm{cd}}^{2}$. From our earlier assumptions, the swept up gas ends up with a velocity $v=v_{\mathrm{cd}}$ (i.e. it is accelerated) and no internal energy. Hence, the final energy per unit mass is $E_{2}=\frac{1}{2} v_{\mathrm{cd}}^{2}$. Therefore the energy radiated away is
$E_{\text {rad }}=E_{1}-E_{2}=\frac{1}{16} v_{\text {cd }}^{2}$ per unit mass. The total energy radiated away over the age of the bubble is:

$E_{\mathrm{rad}}=\int 4 \pi r_{\mathrm{cd}}^{2}\left(\rho_{0} r_{\mathrm{cd}}^{\beta} v_{\mathrm{cd}}\right) \frac{1}{16} v_{\mathrm{cd}}^{2} \mathrm{~d} t$

where the integral is evaluated from $t=0$ to $t=t$. Just as we obtained Eq. (28), we find that

$E_{\mathrm{rad}}=\frac{\pi}{4} \alpha^{10+\lambda} x_{\mathrm{cd}}^{2+\beta} g_{\mathrm{cd}}^{3} \theta^{5+\beta} \dot{E} t$.

Global energy conservation demands that $\dot{E} t=K E_{\mathrm{b}}+$ $I E_{\mathrm{b}}+K E_{\mathrm{sh}}+E_{\mathrm{rad}}$. With the 4 terms on the RHS scaling as $\alpha^{10+\lambda}$, the correct normalization of the solution may be determined.

Finally, we can also calculate the fraction of mass lost by the star over its lifetime that has yet to pass through the inner shock:

$\rho_{\mathrm{sw}}=\frac{M_{\mathrm{usw}}}{M_{\mathrm{w}}}=\alpha^{8+\lambda}\left(\frac{16+3 \lambda}{10+\lambda}\right) \frac{m_{\mathrm{usw}}}{\phi}$.

\subsection{Solution procedure}

Three parameters fully determine the form of a given similarity solution $\left(\lambda, \phi\right.$ and $\left.x_{\text {is }} / x_{\mathrm{cd}}\right)$, whilst a further parameter, $(\theta)$, obtained only after a particular solution has been found, is a measure of the ratio of mass evaporated from the clumps to the mass swept-up from the interclump medium. For bubbles whose evolution is significantly altered by mass-loading, we require that simultaneously both $\phi$ and $\theta$ are low.

The similarity equations were integrated with a fifthorder accurate adaptive step-size Bulirsch-Stoer method using polynomial extrapolation to infinitesimal step size. Once the CD was reached, rescaling was implemented with the relationships in Eq. (20) so that $x_{\mathrm{cd}}=1$. The mass, and kinetic and thermal energies of the bubble were calculated, as were the kinetic energy of the shell and the energy radiated from it. The correct normalization to satisfy global energy conservation was then obtained. Finally, for given values of $\dot{E}, Q$, and $t, x, f, g$ and $h$ may be used to calculate the physical variables $r, \rho, u$, and $\varepsilon$.

\section{Results}

We first consider similarity solutions obtained by Dyson \& de Vries (1972) and Dyson (1973). These earlier works concerned the dynamical effects of a high velocity stellar wind incident on a smooth ambient medium and contain no results for mass-loaded flows. Dyson \& de Vries originally computed the form of the shocked ambient gas behind the forward shock by parameterizing the cooling function, which demanded that the ambient density, $\rho \propto r^{q}$ with $q=-1$. They discovered that the shocked ambient gas cooled rapidly, and that this region was very thin compared with the overall radius of the bubble. Therefore, Dyson (1973) discarded the shocked ambient region in favour of a strong isothermal shock coincident with the 

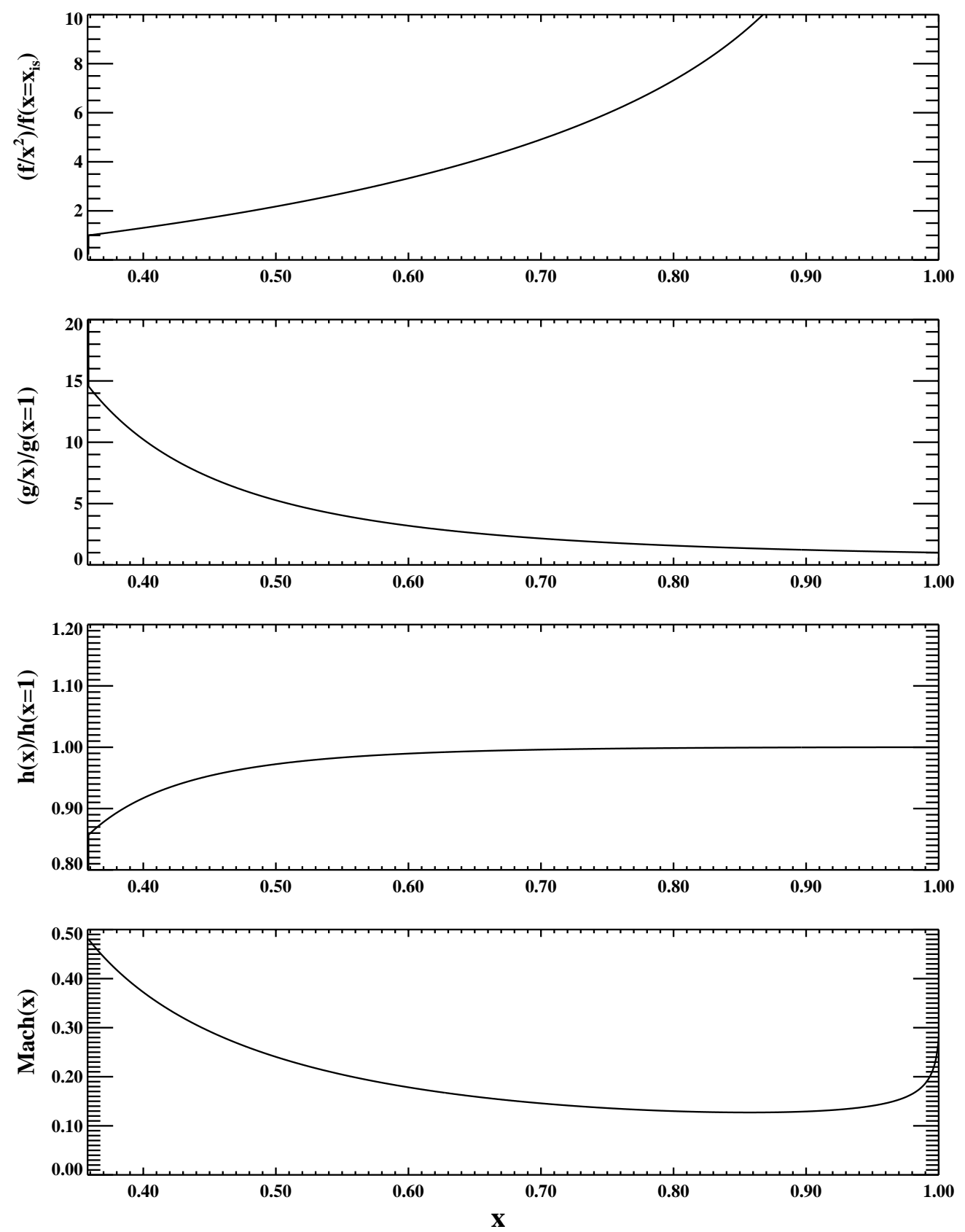

Fig. 2. Results for $\lambda=-3, x_{\text {is }} / x_{\mathrm{cd}}=0.359$, and $\phi=50000$. In the limit of large $\phi$ (i.e. negligible mass-loading), the earlier results of Dyson (1973) are recovered. Specific combinations of the variables are chosen in order to facilitate comparison with results given in Fig. 2 of Dyson (1973). In our work, $f$ is a measure of density, $g$ is a measure of velocity, and $h$ is a measure of the thermal energy density. $\operatorname{Mach}(x)$ is the Mach number of the flow as a function of $x$

contact discontinuity, which relieved the restriction on $q$. A central assumption for both papers was that the velocity of the stellar wind was constant, which led to the stellar mass-loss rate varying as $\dot{M} \propto t^{q+2}$. Thus for an ambient density $\rho \propto r^{-2}$, the energy input by the stellar wind was constant as a function of time. Since in our work the stellar wind velocity is constant if the ambient density falls off as $r^{-2}$ (i.e. $\beta=-2, \lambda=-3$ ), and a constant rate of energy input is a central assumption, we can make a direct comparison between Dyson's (1973) results with those obtained here.
In Fig. 2 we show results for $\lambda=-3$ with negligible mass-loading (large $\phi$ ). We chose specific combinations of the variables in order to facilitate comparison with results given in Fig. 2 of Dyson (1973): agreement is excellent. In Fig. 3 we plot the relative radii (or velocities) of the inner and outer shocks as a function of $g_{*} / g_{\text {cd }}$, the ratio of the stellar wind velocity to the velocity of the contact discontinuity (or outer shock). These calculations were again made with negligible mass-loading. The results for the $\beta=-2$ case may again be directly compared to the earlier results of Dyson (his Fig. 5). In Fig. 3 we also show results for 
Table 1. Properties of solutions with $\lambda=-3(\beta=-2)$. We consider two cases: negligible mass-loading $(\phi=50000)$ and moderate mass-loading $(\phi=1.0)$. Solutions with $\lambda=4$ and $\phi=0.2$ are also listed. From left to right, the columns indicate: i) the ratio of the shock radii, ii- $v$ ) the fraction of energy in the form of thermal energy and kinetic energy in the bubble, kinetic energy in the swept-up shell, and radiated energy, vi) the value of $\theta$, vii) the ratio of mass within the bubble to the total mass lost by the star, viii) the ratio of mass in the swept-up shell to mass in the bubble, ix) the fraction of the mass lost by the star which has yet to pass through the inner shock

\begin{tabular}{lllllllll}
\hline$x_{\mathrm{is}} / x_{\mathrm{cd}}$ & $I E_{\mathrm{b}}$ & $K E_{\mathrm{b}}$ & $K E_{\mathrm{sh}}$ & $E_{\mathrm{rad}}$ & $\theta$ & $\Phi_{\mathrm{b}}$ & $M_{\mathrm{sh}} / M_{\mathrm{b}}$ & $\rho_{\mathrm{sw}}$ \\
\hline$\lambda=-3, \phi=50000$ & & & & & & & & \\
0.34 & 0.45 & 0.03 & 0.47 & 0.06 & 1286 & 1.075 & 162 & 0.018 \\
0.49 & 0.40 & 0.09 & 0.46 & 0.06 & 603 & 1.068 & 35.6 & 0.053 \\
0.69 & 0.26 & 0.29 & 0.40 & 0.05 & 247 & 1.052 & 5.8 & 0.175 \\
0.89 & 0.05 & 0.74 & 0.19 & 0.02 & 86 & 1.046 & 0.60 & 0.505 \\
\hline$\lambda=-3, \phi=1.0$ & & & & & & & & \\
0.12 & 0.47 & 0.002 & 0.47 & 0.06 & 45 & 2.64 & 4098 & 0.0008 \\
0.26 & 0.46 & 0.01 & 0.47 & 0.06 & 9.7 & 2.26 & 219 & 0.0079 \\
0.53 & 0.36 & 0.15 & 0.44 & 0.05 & 1.97 & 1.63 & 12.3 & 0.079 \\
0.73 & 0.21 & 0.40 & 0.35 & 0.04 & 0.85 & 1.20 & 2.86 & 0.232 \\
0.95 & 0.01 & 0.92 & 0.05 & 0.01 & 0.20 & 1.00 & 0.11 & 0.724 \\
\hline$\lambda=4, \phi=0.2$ & & & & & & & & \\
0.18 & 0.70 & 0.005 & 0.24 & 0.06 & 15.3 & 1.26 & 4151 & 0.0079 \\
0.37 & 0.66 & 0.05 & 0.23 & 0.06 & 3.24 & 1.36 & 171 & 0.077 \\
0.42 & 0.49 & 0.38 & 0.11 & 0.03 & 0.66 & 7.87 & 0.71 & 0.303 \\
\hline
\end{tabular}

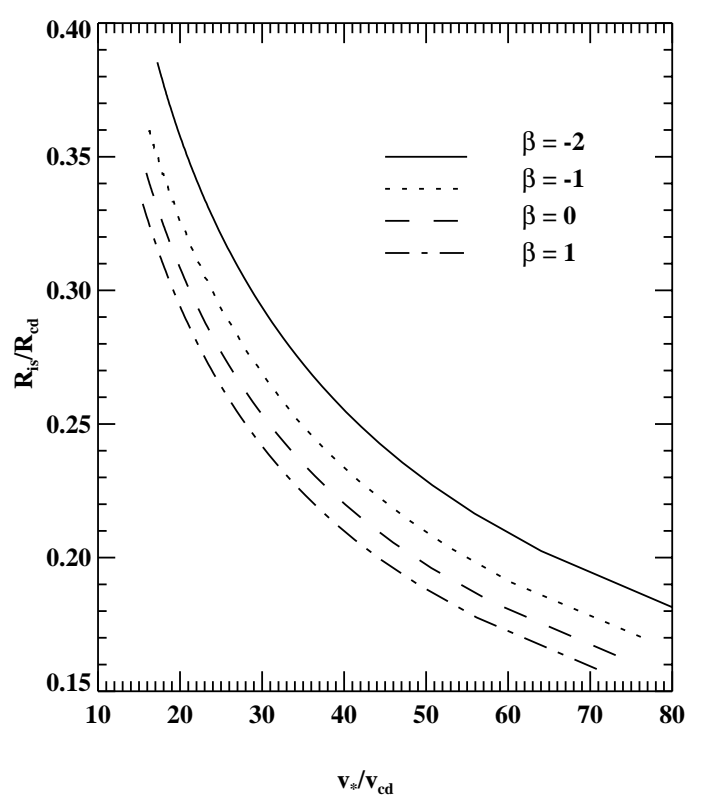

Fig. 3. The ratio of the radii of the inner and outer shocks as a function of $g_{*} / g_{\mathrm{cd}}$ for cases with negligible mass loading. For $\beta=-2$ the earlier results of Dyson (1973) are again recovered (cf. his Fig. 3). For $\beta \neq-2$ the velocity of the stellar wind is a function of time, so these curves cannot be compared to those in Dyson's Fig. 3. For $\beta=-2,-1,0,1$, the corresponding values of $\lambda$ are $-3,-\frac{2}{3}, \frac{5}{3}, 4$

other values of $\beta$. These cannot be compared to Dyson's earlier results because when $\beta \neq-2$ the stellar wind velocity is no longer constant with time. We find that as the value of $\beta$ increases (which also corresponds to the massloading becoming increasingly dominant at large radii), the shocked region broadens for a given ratio of $g_{*} / g_{\mathrm{cd}}$.

In Fig. 4 we present results for a case in which massloading dominates the evolution of the bubble. In this particular case, $\lambda=4, \phi=0.2, x_{\mathrm{is}}=0.403 x_{\mathrm{cd}}$, and $\theta=0.62$. The bubble mass is over 8 times higher than the total mass lost by the star, and almost twice the mass of the ambient medium swept up into the thin shell. 30 per cent of the mass lost by the star has still to pass through the inner shock. The high degree of mass-loading increases the post-shock density, and dramatically reduces the temperature in the post-shock region as more and more mass is evaporated. This reduces the sound speed, and there is eventually a sonic transition close to the CD as the flow becomes supersonic in the frame of the clumps.

Values of several parameters calculated from solutions with $\lambda=-3$ are given in Table 1 . If the mass-loading is negligible (illustrated in the table by $\phi=50000$ ), we find the following: i) as the ratio of $x_{\text {is }} / x_{\mathrm{cd}}$ increases, the fraction of energy contained in the form of thermal energy in the bubble and that in the form of kinetic energy of the shell decreases, whilst that in the form of kinetic energy of the bubble increases. The fraction of energy radiated away by the shocked interclump gas stays relatively constant, and is always a small part ( $<6$ per cent) of the total mechanical energy of the stellar wind over its lifetime. ii) The mass contained in the bubble is larger than the mass of the swept-up shell only when the relative radius of the inner shock is large. iii) For small $x_{\text {is }} / x_{\mathrm{cd}}$, about 90 per cent of 

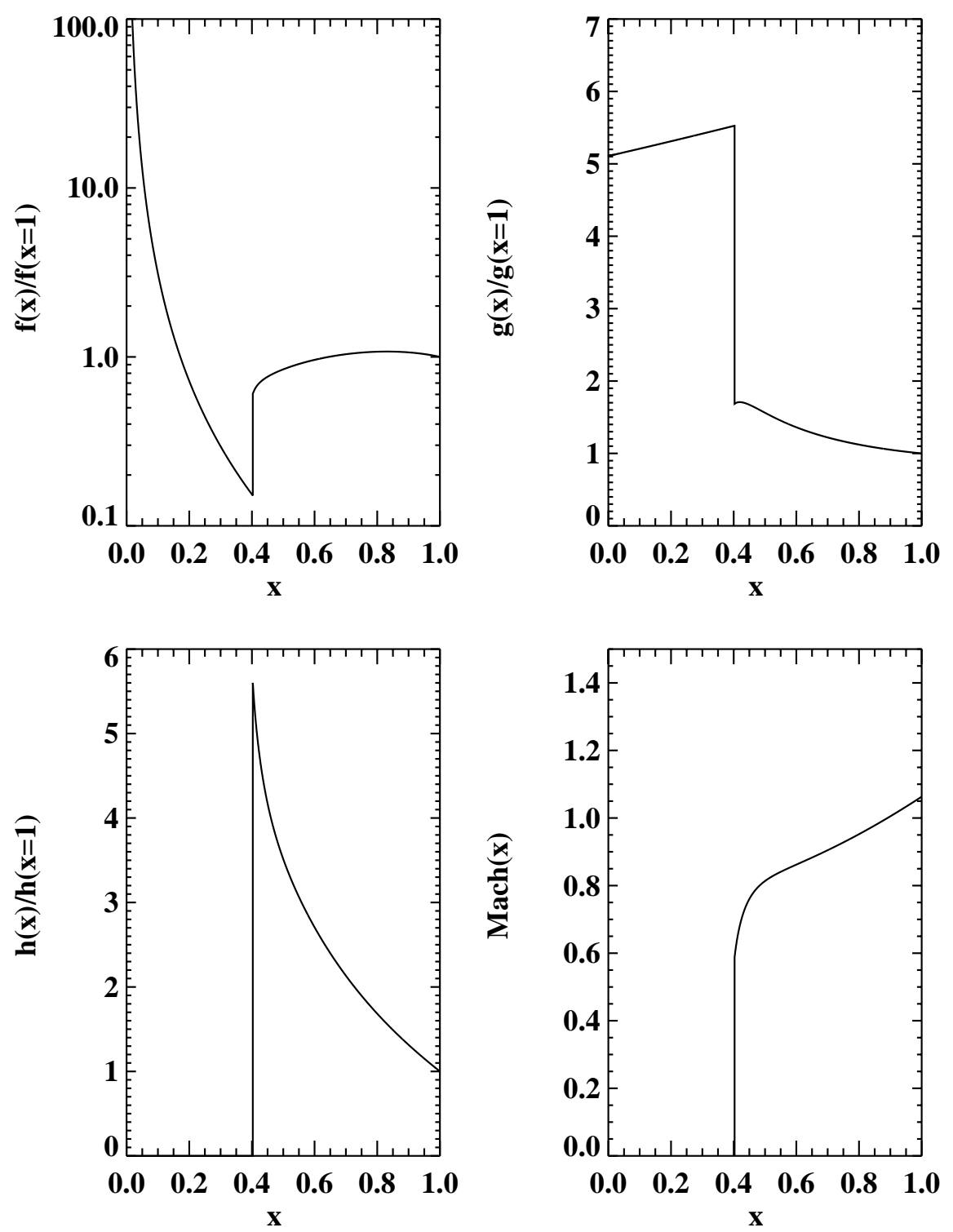

Fig. 4. Results for a bubble with significant mass-loading. This particular solution is for $\lambda=4, \phi=0.199$, and $x_{\text {is }}=0.403 x_{\mathrm{cd}}$. The bubble mass is 8.4 times the total mass lost by the star. The ratio of the swept-up mass to the bubble mass is $M_{\mathrm{sh}} / M_{\mathrm{b}}=0.54$

the total mechanical energy of the stellar wind is split approximately evenly between the thermal energy contained in the SSW region and the kinetic energy of the swept up shell. As $x_{\text {is }} / x_{\text {cd }}$ increases, these fractions decrease, whilst the kinetic energy of the gas inside the bubble increases, until it eventually may exceed 90 per cent of the total energy. iv) The kinetic energy of the swept-up shell is always as great or greater than the thermal energy of the bubble (this is also true for the solutions in Table 1 with $\lambda=-3$, $\phi=1.0)$.

When we set $\phi=1.0$, the bubble is just beginning to become mass-loaded for small values of $x_{\mathrm{is}} / x_{\mathrm{cd}}$. However, for $\lambda=-3$, the swept-up mass dominates the bubble mass in this regime, as can be seen from the values in Table 1 . We find that solutions for which both $\Phi_{\mathrm{b}}$ is large and $M_{\mathrm{sh}} / M_{\mathrm{b}}$ is small exist only if $\lambda \gtrsim 4$. Solutions for $\lambda=4(\beta=1)$ and $\phi=0.2$ are illustrated in Fig. 5 . The solution with $x_{\text {is }} / x_{\mathrm{cd}}=0.420$ is strongly mass-loaded, and in contrast to the solutions with $\lambda=-3$ the mass of the swept up shell is also less than $M_{\mathrm{b}}$. We note that when $x_{\text {is }} / x_{\text {cd }}$ is varied, notable differences between the profiles of $f, g$, and $h$ occur in Fig. 5. For instance, when the bubble is only weakly mass-loaded the density at the CD is smaller than immediately after the inner shock. However, once the bubble is more strongly mass-loaded the density continuously rises from the inner shock to the CD. Other differences include less deceleration of the flow, a sharp fall in the temperature (instead of a gradual rise), and a rise in the Mach number. Finally, when the bubble becomes strongly mass-loaded, the fractional distribution of the total energy between $I E_{\mathrm{b}}, K E_{\mathrm{b}}, K E_{\mathrm{sh}}$, and $E_{\mathrm{rad}}$ for a given $x_{\mathrm{is}} / x_{\mathrm{cd}}$ ratio is substantially different than when the 

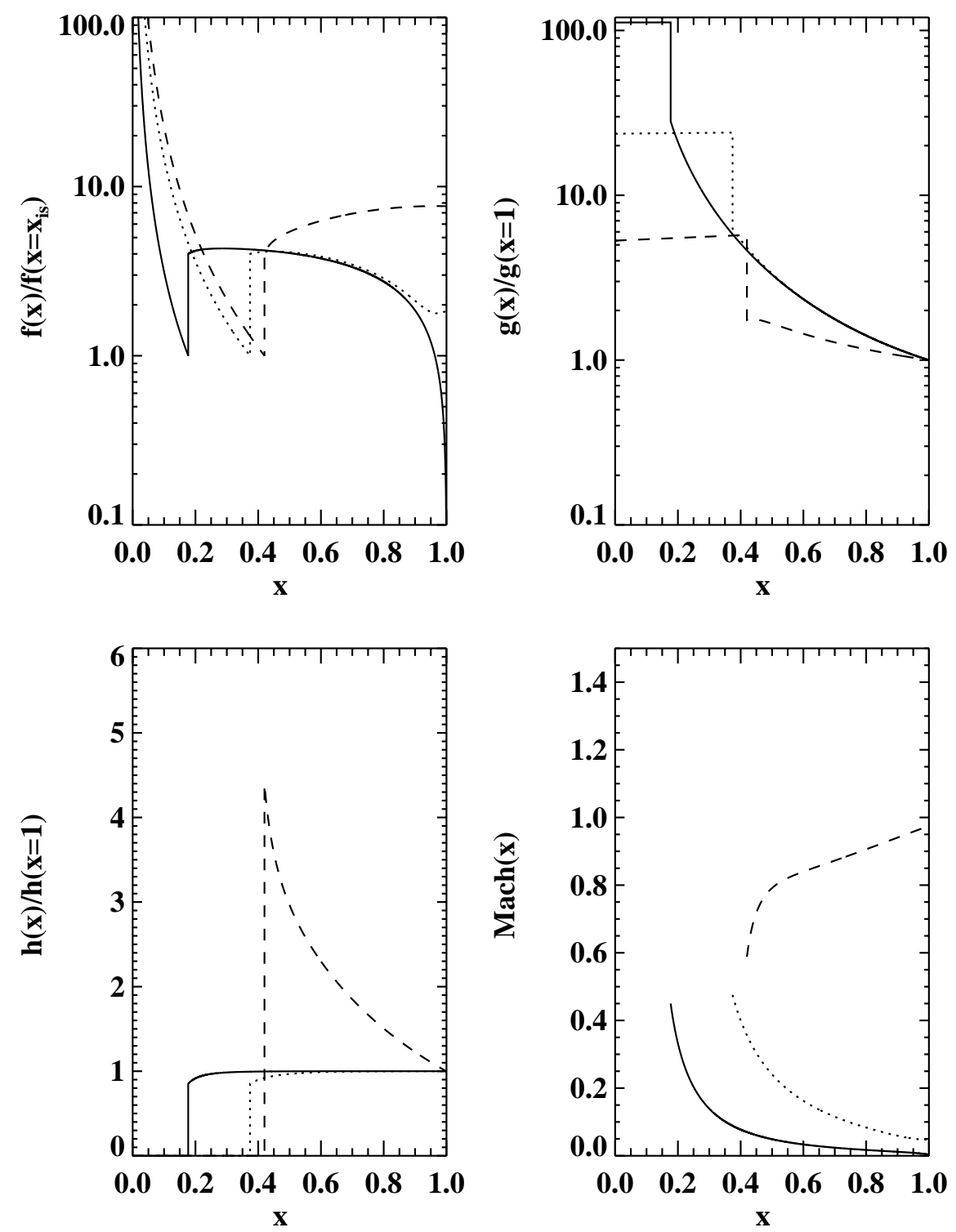

Fig. 5. Results for bubbles in which the radial dependence of the mass-loading is $\propto r^{4}$. Three solutions with different values of $x_{\text {is }} / x_{\mathrm{cd}}$ are shown: $0.177,0.374$, and 0.420 (solid, dotted, and dashed lines). $\Phi_{\mathrm{b}}$ is equal to $1.26,1.36$ and 7.86 respectively, whilst $M_{\mathrm{sh}} / M_{\mathrm{b}}$ is equal to $4151,171,0.71$ respectively. For ease of comparison, $f$ is scaled such that its pre-shock value is unity in each case

mass-loading is negligible. This is hardly surprising given the change seen in the profiles of the flow variables.

An important finding is that for a given $\lambda$ and ratio $x_{\text {is }} / x_{\mathrm{cd}}$, there exists a minimum value of $\phi$ at which we can find a solution. In other words, there is a limit to the maximum mass-loading which the bubble can undergo. In Fig. 6 we show the value of $\phi_{\min }$ for various values of $x_{\text {is }} / x_{\mathrm{cd}}$ and $\beta$. This finding has the corollary that for a given value of $\phi$ there is a maximum value of $x_{\text {is }} / x_{\mathrm{cd}}$. The numerical reason that we are unable to obtain solutions with $\phi<\phi_{\min }$ is that the denominator of the derivatives from Eqs. (8-10) approaches zero without the numerators doing likewise. The physical reason is that there is a straightforward feedback mechanism limiting the amount of mass-loading that may take place: as mass from the clumps is evaporated, the temperature of the shocked region falls, which reduces the rate at which further mass can be evaporated. Similarly, if the massloading were suddenly inhibited, the temperature of the shocked region would rise, leading to an increase in the evaporation rate. There is no reason to believe that actual wind bubbles would be at this limit because the degree of mass-loading simply depends on the number of clumps present and their individual mass injection rates (which in our model is characterized by the value of $\phi_{\mathrm{b}}$ ). For a given mass, the number density of clumps, $n_{\mathrm{c}} \propto r_{\mathrm{c}}^{-3}$ where $r_{\mathrm{c}}$ is the clump radius, whilst the mass injection rate from each clump $\dot{m}_{\mathrm{c}} \propto r_{\mathrm{c}}$. Thus we can only comment that for small clumps, it is more likely that this maximum mass-loading limit will actually occur. 

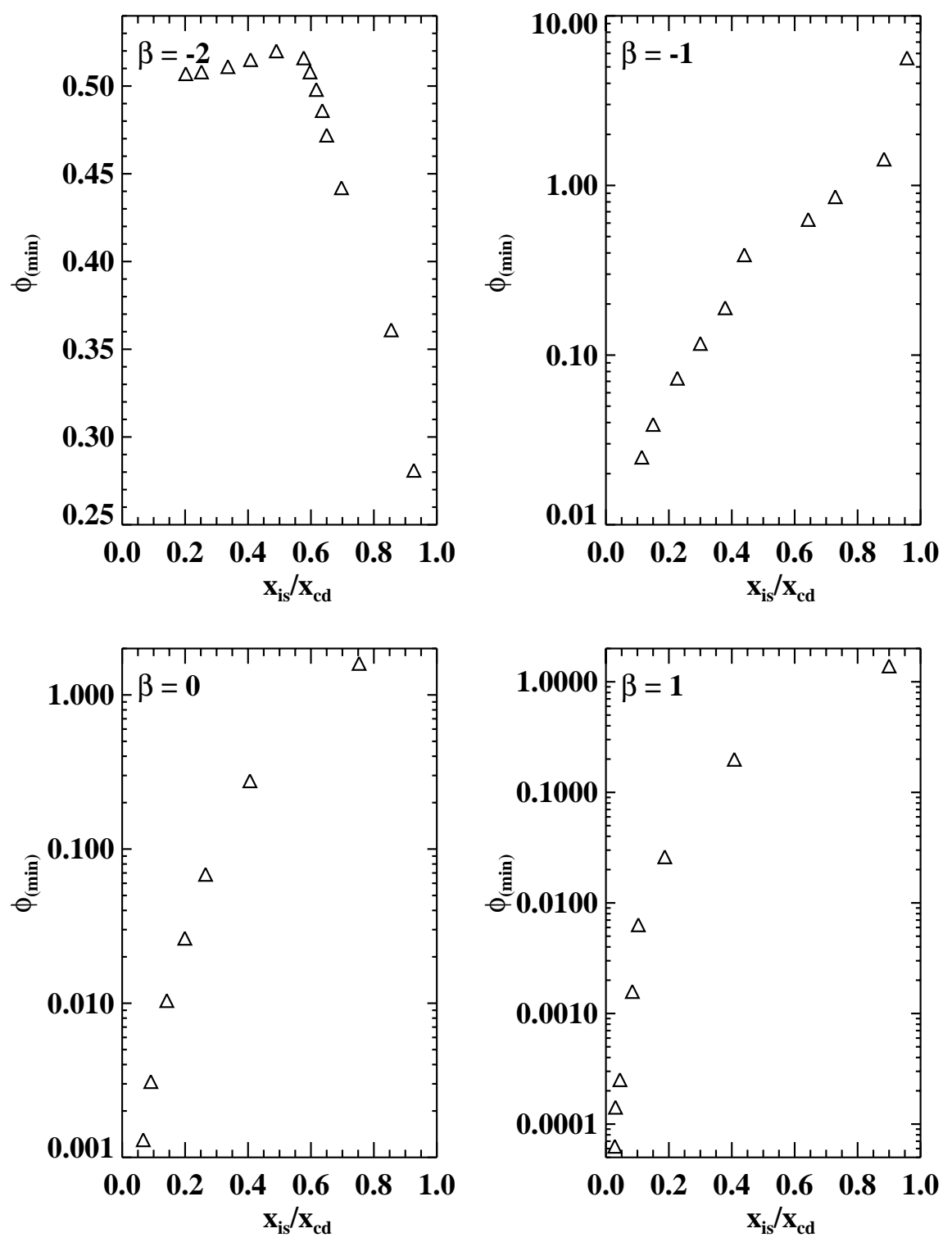

Fig. 6. The minimum value of $\phi$ (i.e. corresponding to the maximum possible mass-loading) for which solutions could be found, as a function of $x_{\mathrm{is}} / x_{\mathrm{cd}}$ and $\beta$ (or, alternatively $\lambda$ )

We further note that the radial profile of the Mach number of the flow relative to the clumps can take many different forms, depending on the input parameters used. In Fig. 7 we show several examples. The profile of the Mach number is influenced by the fact that mass-loading tends to drive the Mach number towards unity, whilst the spherical divergence tends to drive it away from unity (cf. Hartquist et al. 1986). Panel a) is for a case in which massloading is small. In this case the divergence term wins, and drives the Mach number towards zero. Panel b) is for a similar situation, but in this case because the inner shock is very close to the CD, the initial postshock Mach number is greater than unity so the divergence term drives it towards infinity. In panel c) we see that the divergence is initially dominant, but as the flow approaches the CD the Mach number is driven back towards unity. However, this behaviour is not due to the flow mass-loading (although
$\left.\Phi_{\mathrm{b}}=2.64\right)$, but is rather because the density increases near the CD whilst the pressure remains constant, producing a decrease in the sound speed (the same effect occurs for the flow shown in Fig. 2 which has no mass-loading). The same situation occurs in panel d) where the fall in sound speed is great enough to drive the flow through a sonic transition. The flow corresponding to panel e) is very similar to that associated with panel d) except we now have two sonic transitions due to the initial postshock flow being supersonic with respect to the clumps. In plot $\mathrm{f})$ the flow is significantly mass-loaded $\left(\Phi_{\mathrm{b}}=7.86\right)$, and the mass-loading term dominates the divergence term such that the Mach number is driven towards unity.

Finally, we have calculated profiles of the X-ray emissivity as a function of radius (see Fig. 8). We assume that the emissivity $\Lambda \propto n^{2} T^{-1 / 2}$, which is a good approximation over the temperature range $510^{5} \mathrm{~K} \lesssim T \lesssim 510^{7} \mathrm{~K}$ 

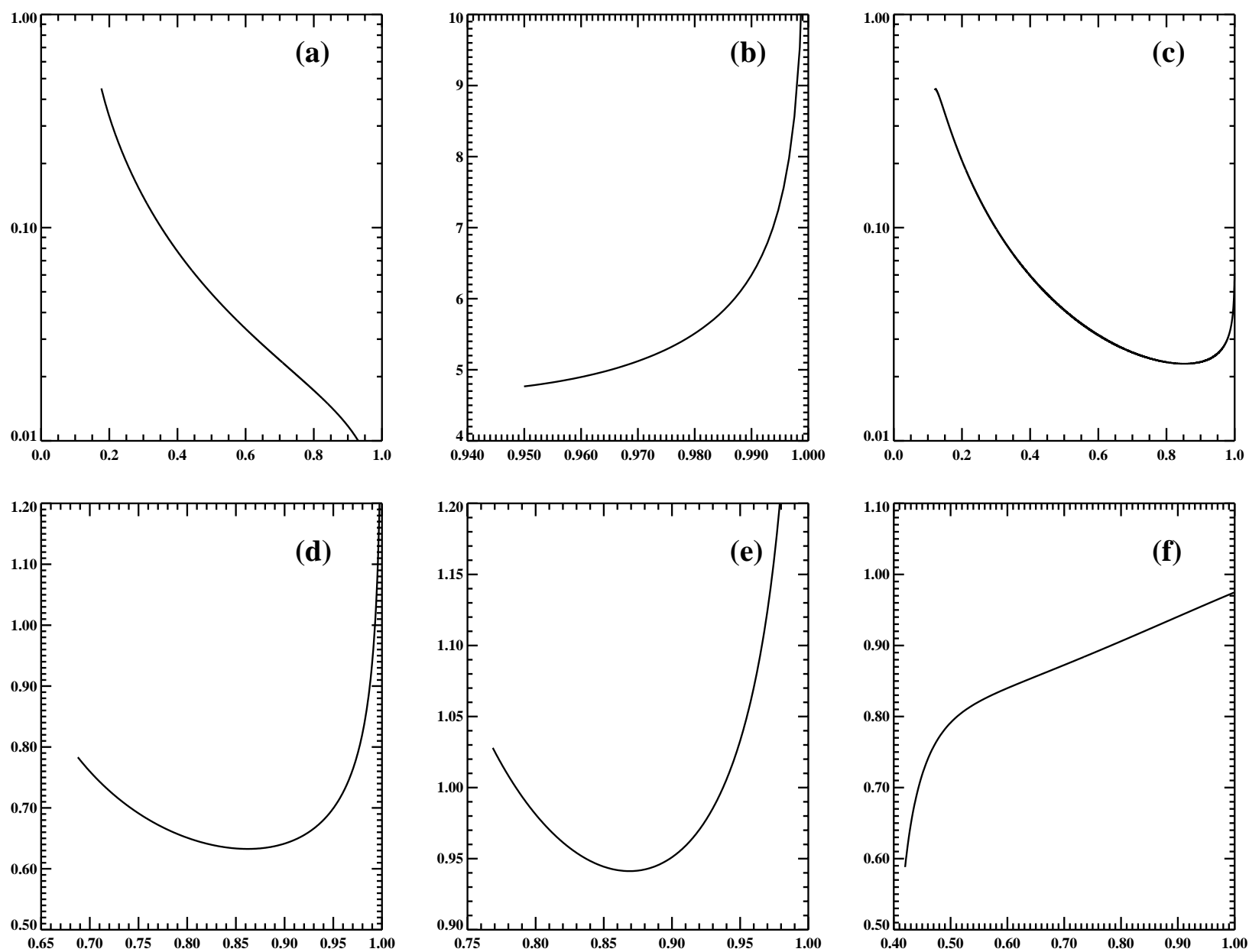

Fig. 7. The Mach number as a function of $x$ for a number of different solutions. With respect to the clumps, the shocked region can be either entirely subsonic, entirely supersonic, or have up to two sonic points. The corresponding solution shown in each panel is: a) $\lambda=4, \phi=0.2, x_{\mathrm{is}} / x_{\mathrm{cd}}=0.177$; b) $\left.\left.\lambda=-3, \phi=1.0, x_{\mathrm{is}} / x_{\mathrm{cd}}=0.950 ; \mathbf{c}\right) \lambda=-3, \phi=1.0, x_{\mathrm{is}} / x_{\mathrm{cd}}=0.120 ; \mathbf{d}\right)$ $\left.\left.\lambda=-3, \phi=50,000, x_{\mathrm{is}} / x_{\mathrm{cd}}=0.688 ; \mathbf{e}\right) \lambda=-3, \phi=50,000, x_{\mathrm{is}} / x_{\mathrm{cd}}=1.301 ; \mathbf{f}\right) \lambda=4, \phi=0.2, x_{\mathrm{is}} / x_{\mathrm{cd}}=0.420$

(cf. Kahn 1976). Also plotted in Fig. 8 are the radial temperature profiles. A bubble un-affected by mass-loading expanding into a surrounding medium with an $r^{-2}$ profile of density has a higher central temperature but a lower central emissivity than at its limb. Conversely, if it is expanding into a medium with an $r^{1}$ profile of density, the situation is reversed, as the central temperature is lower and the central emissivity is higher than at its limb. If the bubble is mass-loaded, the general trend is for a reduction in the central emissivity and an increase in the central temperature relative to the limb. To date, the only stellar wind bubbles which have been successfully observed in X-rays are two Wolf-Rayet ring nebulae, NGC 6888 (e.g. Wrigge et al. 1998) and S308 (Wrigge 1999). Both have Xray luminosities lower than expected from the standard model (Weaver et al. 1977). This discrepancy is normally attributed to two possibilities: either conductive evaporation of gas from the cold dense outer shell into the bubble interior, or enhanced cooling resulting from high metallicities in the cooling gas (see MacLow 2000). It is not surprising, therefore, that our mass-loading simulations indicate that evaporation from clumps within the bubble may also be compatible with current observations. The latest X-ray satellites, Chandra and XMM, should provide significantly improved observations which may enable us to distinguish between these competing mechanisms. One might also expect that they may allow discrimination between a mass-loaded bubble expanding into an ambient medium with $\rho \propto r^{1}$ and a bubble unaffected by massloading expanding into a medium with $\rho \propto r^{-2}$.

\section{Summary}

We have investigated the evolution of a mass-loaded wind blown bubble with a constant rate of ejection of mechanical energy from a central stellar wind. Mass-loading is assumed to occur by the conductively induced evaporation of clumps in the region of shocked stellar wind. A central assumption is that the shocked interclump medium rapidly cools to a dense, negligibly thin, shell through which the clumps can pass undisturbed. The requirement that the solution be self-similar imposes a link between the radial variation of the interclump density $\left(\rho \propto r^{\beta}\right)$ and 

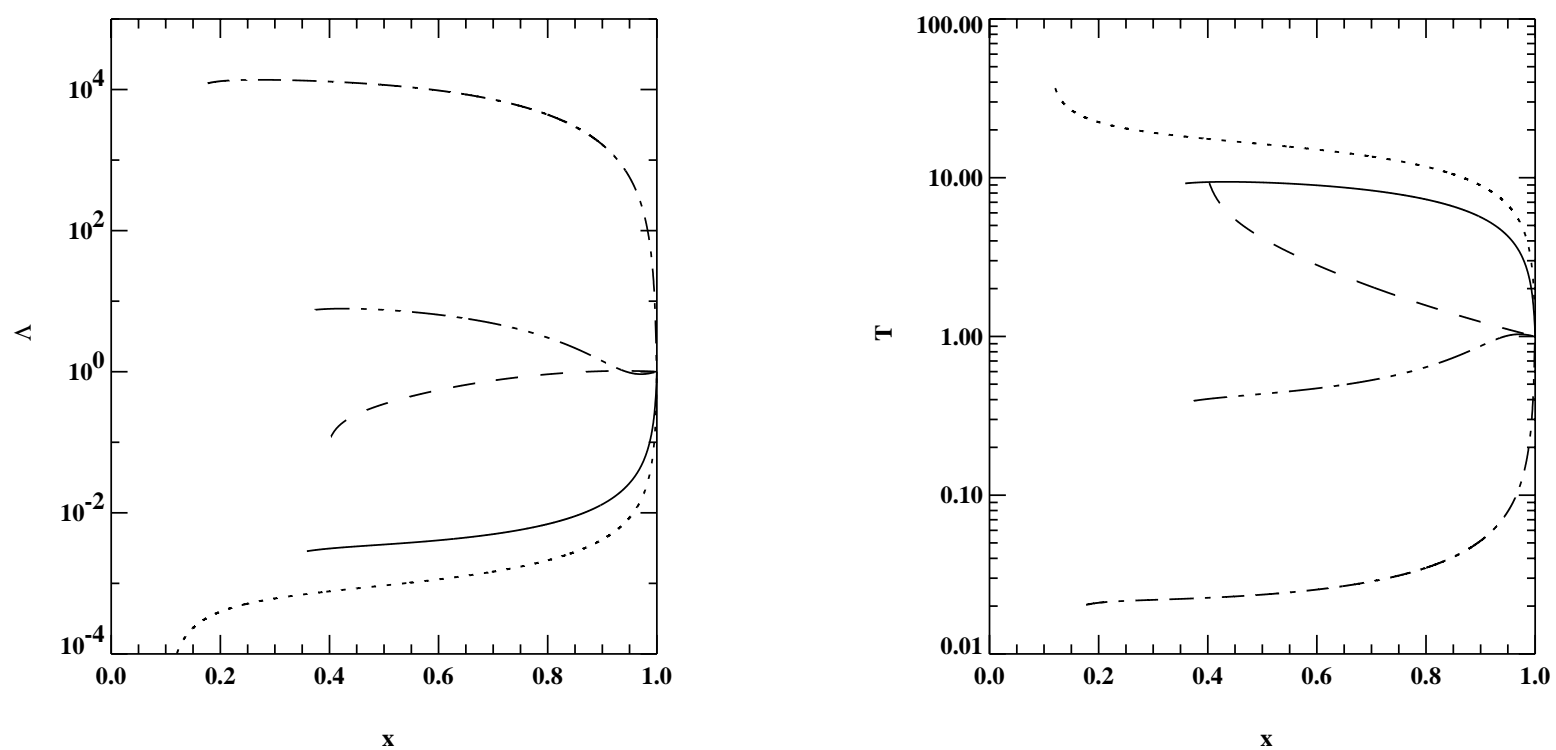

Fig. 8. Radial profiles of emissivity per unit volume and temperature, normalized to values of 1.0 at the limb, for a number of the simulations previously discussed. These are $\lambda=-3, \Phi_{\mathrm{b}}=1.0$ (solid); $\lambda=-3, \Phi_{\mathrm{b}}=2.64$ (dots); $\lambda=4, \Phi_{\mathrm{b}}=1.26$ (dashes); $\lambda=4, \Phi_{\mathrm{b}}=1.36$ (dot-dash); $\lambda=4, \Phi_{\mathrm{b}}=7.87$ (chain-dot-dash). The general trend is for a reduction in the central emissivity and an increase in the central temperature with increased mass loading

the mass-loading from the clumps $\left(\dot{\rho} \propto r^{\lambda}\right)$ which forces $\lambda=(5+7 \beta) / 3$. We first reproduced solutions with negligible mass-loading and $\beta=-2$, which we compared with results obtained by Dyson (1973). Excellent agreement for the structure of the bubble was found. We also found that the ratio of the radii (velocities) of the inner and outer shocks decreases with increasing $\beta$ for a given ratio of the current stellar wind velocity to the $\mathrm{CD}$ velocity.

We then investigated the changes in the structure of the bubble for different values of $\lambda, \phi$, and $x_{\mathrm{is}} / x_{\mathrm{cd}}$. The central conclusions are:

- Substantial mass-loading of the wind-blown bubble can occur over a wide range of $\lambda$. However, to additionally satisfy the requirement that the bubble mass is larger than the mass of the swept-up shell, large values of $\lambda$ are needed $(\lambda \gtrsim 4)$;

- The profiles of the flow variables are significantly altered under conditions of large mass-loading. In particular, the average density of the shocked region is larger, the deceleration of the flow is shallower, and the temperature of the shocked wind rapidly decreases;

- The Mach number of the shocked stellar wind relative to the clumps which are injecting the mass can take several different forms. The shocked region can be either entirely subsonic, entirely supersonic, or have one or sometimes two sonic points;

- For a given $\lambda$ and ratio of the shock radii, there exists a maximum mass-loading that can occur;

- Mass-loading tends to reduce the emissivity in the interior of the bubble relative to its limb, whilst simultaneously increasing the central temperature relative to the limb temperature.

Acknowledgements. JMP would like to thank PPARC for the funding of a PDRA position, and Sam Falle and Rob Coker for helpful discussions. We would also like to thank an anonymous referee whose suggestions improved this paper.

\section{References}

Arthur, S. J., Dyson, J. E., \& Hartquist, T. W. 1993, MNRAS, 261,425

Arthur, S. J., Dyson, J. E., \& Hartquist, T. W. 1994, MNRAS, 269, 1117

Arthur, S. J., Henney, W. J., \& Dyson, J. E. 1996, MNRAS, 313,897

Chièze, J. P., \& Lazareff, B. 1981, A\&A, 95, 194

Cowie, L. L., \& McKee, C. F. 1977, ApJ, 211, 135

Cowie, L. L., McKee, C. F., \& Ostriker, J. P. 1981, 247, 908

Dyson, J. E. 1973, A\&A, 23, 381

Dyson, J. E., \& de Vries, J. 1972, A\&A, 20, 223

Dyson, J. E., \& Hartquist, T. W. 1987, MNRAS, 228, 453

Hartquist, T. W., Dyson, J. E., Pettini, M., \& Smith, L. J. 1986, MNRAS, 221, 715

Kahn, F. D. 1976, A\&A, 50, 145

MacLow, M.-M. 2000, RMxAc, 9, 273

McKee, C. F., \& Cowie, L. L. 1977, ApJ, 215, 213

McKee, C. F., \& Ostriker, J. P. 1977, ApJ, 218, 148

Meaburn, J., Nicholson, R. A., Bryce, M., Dyson, J. E., \& Walsh, J. R. 1991, MNRAS, 252, 535

Meaburn, J., Clayton, C. A., Bryce, M., et al. 1998, MNRAS, 294, 201

Smith, L. J., Pettini, M., Dyson, J. E., \& Hartquist, T. W. 1984, MNRAS, 211, 679

Weaver, R., McCray, R., Castor, J., Shapiro, P., \& Moore, R. 1977, ApJ, 218, 377 (erratum 220, 742)

Williams, R. J. R., Dyson, J. E., \& Hartquist, T. W. 1999, A\&A, 344, 675

Williams, R. J. R., Hartquist, T. W., \& Dyson, J. E. 1995, ApJ, 446, 759

Wrigge, M. 1999, A\&A, 343, 599

Wrigge, M., Chu, Y.-H., Magnier, E.A., \& Kamata, Y. 1998, LNP, 506, 425 